FACTA UNIVERSITATIS

Series: Mechanical Engineering Vol. 16, N² 2, 2018, pp. 139 - 155

https://doi.org/10.22190/FUME180420016M

Original scientific paper

\title{
A CLOUD-BASED EXPERT SYSTEM FOR SYNTHESIS AND EVOLUTIONARY OPTIMIZATION OF PLANAR LINKAGES
}

UDC 621.7

\author{
Rosen Mitrev ${ }^{1}$, Boris Tudjarov ${ }^{2}$, Todor Todorov ${ }^{3}$ \\ ${ }^{1}$ Technical University, Mechanical Engineering Faculty, Sofia, Bulgaria \\ ${ }^{2}$ Technical University, Faculty of Computer Systems and Technologies, Sofia, Bulgaria \\ ${ }^{3}$ Technical University, Faculty of Industrial Technology, Sofia, Bulgaria
}

\begin{abstract}
The present paper introduces a cloud-based expert system for synthesis and evolutionary optimization of planar linkages. The kinematic structure of the linkage is composed by the modular approach based on Assur's groups. The dyads are represented as functional blocks with input and output variables. The applied approach for obtaining the geometrical relationships between the input and the output variables of the dyads is based on the use of homogeneous transformation matrices. The developed software system allows a dimensional synthesis of planar linkages by using genetic optimization algorithms. One feature is remote creation of the models of genetic algorithms as well as the receiving of the results by means of a user-friendly interface. By exploiting the application, the user can produce and edit the initial information about the synthesized or optimized linkage; thus he can receive the calculation results as a web page and/or as MS Excel file. An additional mutation of the best chromosome genes by scanning of every gene within its searching space improves the optimal solution. The analyzed numerical case studies show the applicability of the developed software system for mechanism analysis, synthesis and optimization. Because the number of genes is not limited, the linkages with a very big number of design variables can be synthesized by exploiting the developed approach.
\end{abstract}

Key Words: Planar Linkage, Assur's Groups, Genetic Algorithms, Expert System

\section{INTRODUCTION}

In the past two decades, along with the classical graphical and analytical techniques $[1,2]$, there has been an increasing interest in the use of computer technologies for

Received April 20, 2018 / Accepted May 28, 2018

Corresponding author: Rosen Mitrev

Technical University, Mechanical Engineering faculty, Kliment Ohridski 8 blvd., Sofia, Bulgaria

E-mail: rosenm@tu-sofia.bg 
modeling and simulation of machines and mechanisms in education and engineering practice [3,4]. An easy applied and widely used approach is the modeling by way of special or general-purpose mechanical dynamics and kinematics software with different functionality realized on different platforms. Some programs are fully interactive, offering an easy-to-use environment [5-7] and possessing modules for preprocessing, numerical analysis and results post-processing. Simultaneously with undeniable advantages in its use, this type of software has some significant drawbacks: it is usually high-priced, the obtained results are limited to the software capabilities, equations of motion are embedded in the program and cannot be previewed by the user, and, finally yet importantly - it does not allow further development of algorithms by the user. Software systems where an active involvement of the user in the mechanism simulation model development is required are becoming increasingly popular. This type of software is based primarily on the algorithmic programming languages. For example, the $\mathrm{C} / \mathrm{C}++$ compatible object-oriented software [8] provides for a possibility of realizing independent applications in a Web environment and capabilities for performing a kinematic and dynamic analysis of a variety of mechanisms as well as synthesizing mechanisms with predefined properties. Other applications [9-11] are entirely Web-based and platform independent client-server systems, exploiting the advantages of the network computing. Typically, in this case, standard feature rich libraries for mechanism visualization, animation and results plotting are available. In some cases, the software systems are equipped with modules for mechanism type or dimensional synthesis, based on analytical or numerical methods [12].

A widely used approach, considerably facilitating the mechanisms creation, analysis and synthesis, is the modular approach [13, 14], which uses predefined blocks and subroutines for composing mechanisms with arbitrary complexity. During the realization of the modular kinematics it is possible to use different modeling approaches and philosophies. Such systems as OpenModelica [15] use graphical blocks to compose the mechanism kinematical structure while others use a collection of software subroutines for kinematic simulation, written in general-purpose [16] or computer algebra programming languages [17].

Despite the presence of a vast number of software systems, the capabilities of the modular approach combined with optimization for the purpose of mechanism synthesis in the Web environment are not used enough. The paper presents an open cloud-based expert system for dimensional synthesis and optimization of planar linkages based on the theory of Assur's groups and genetic algorithms. The modular approach applied to the building of the linkages allows for their fast creation, modification, analysis, synthesis and optimization in a user-friendly cloud-based Internet environment, fully exploiting the benefits of the network computing. This paper is organized as follows: In Section 1 papers dealing with mechanisms different modeling approaches and philosophies are analyzed. Section 2 is devoted to the derivation of the Assur's groups position, velocity and acceleration kinematic equations. Section 3 gives the structure of the developed cloud-based expert system for synthesis and optimization of planar linkages. Section 4 presents the description and discussion of the synthesis of four-bar and six-bar planar linkages. Section 5 represents a short conclusion. 


\section{ASSUR's GROUPS KINEMATIC EQUATIONS}

The idea of decomposition of the mechanisms into Assur's groups is not new. At the beginning of the $20^{\text {th }}$ century, the Russian scientist Leonid Assur developed a method of composing planar mechanisms of any complexity by the sequential appending of fundamental kinematic chains, which were later named Assur's groups. The number of links $n$ and the number of the fifth class pairs $p_{5}$ in the Assur's groups are related by the following equation:

$$
p_{5}=\frac{3}{2} n
$$

Because $n$ and $p_{5}$ must be integer numbers, the first possible solution of the above equation is $n=2$ and $p_{5}=3$, i.e. the simplest fundamental kinematical chain consists of two links and three fifth class kinematical pairs. Internal kinematical pair of the group connects the two group links to each other and two external pairs connect the group to a driver link, to the other groups or to the ground. This simplest type of group is often called a binary group or dyad. Each of the dyads has zero mobility and their appending to the mechanism does not change the DOF (degree of freedom) of the whole mechanism. One can distinguish the following types of dyads: RRR, RRT, RTR, TRT, RTT, where R denotes rotational one DOF pair and $T$ - translational one DOF pair. The RRR dyad is called Assur's group of the first type. The rest of the dyads are created by replacement of the rotational with the translational pairs. The vast majority of the industrial linkages can be created by the combination of one or more dyads with the addition of one or more rotational or translational driving links.

A substantial advantage of using dyads is the possibility to perform an independent kinematical analysis of each group and then compose a solution for the whole mechanism as a combination of partial solutions for different dyads. The primary goal of the solution is to describe the motion of the dyad according to the referential coordinate frame.

Let us demonstrate the derivation and analytical solution of the kinematic equations for RRR dyad by using rotation and homogeneous transformation matrices, widely used in robotics. In order to specify the position of the dyad pairs, it is necessary to define their Cartesian coordinates in the fixed space reference coordinate system $\left\{x_{0} y_{0}\right\}$. To each rigid link of the dyad is attached a fixed coordinate frame $\left\{x_{k} y_{k}\right\}, k=1,2$. The pose of the link is described by the position of its frame origin and the orientation of its $x$-axis according to the reference coordinate system. Fig. 1 shows the geometrical relationships between the global and local representations of the dyad specific points. The orientation of link $k$ is specified by the angle of rotation $\varphi_{k}$ of link $x_{k}$ axis relative to $x_{0}$ axis of the reference coordinate system. Angle $\varphi_{k}$ is considered as positive if the rotation of $x_{k}$ axis according to positive $x_{0}$ axis is counterclockwise. To point $O_{3}$ is attached a local coordinate system $\left\{x_{e} y_{e}\right\}$ parallel to the reference frame.

As the input for the position analysis of the RRR dyad are used coordinates $\left(x_{1}, y_{1}\right)$ and $\left(x_{3}, y_{3}\right)$ of two external rotational pairs and lengths $L_{1}$ and $L_{2}$ of the links. As the output are received coordinates $\left(x_{2}, y_{2}\right)$ of the internal pair and angles $\varphi_{1}$ and $\varphi_{2}$. Thus, a dyad can be considered as a functional block which has input and output variables, related by known kinematical relationships. This type of dyad representation allows creating subprograms or modules for each dyad type and utilizing them as building blocks when creating linkages. 


\subsection{Formulation of the position equations}

Formulation of the position equations constitutes the most difficult part of the kinematical analysis. Over the years, various approaches for formulation and analytical or numerical solution of the position equations are used [18-22]. A method to establish the geometrical relationships between coordinates of the group external pairs $O_{1}$ and $O_{3}$ is by using homogeneous transformation matrices. They represent a mapping from one frame to another:

$$
{ }_{A}^{B} \mathbf{T}(\varphi, x, y)_{3 \times 3}=\left[\begin{array}{cc}
{ }_{A}^{B} \mathbf{R}(\varphi)_{2 \times 2} & { }_{A}^{B} \mathbf{P}(x, y)_{2 \times 1} \\
\mathbf{0}_{1 \times 2} & 1
\end{array}\right]
$$

where by ${ }_{A}^{B} \mathbf{R}(\varphi)$ is denoted the rotation matrix between two arbitrary coordinate systems $A$ and $B$ :

$$
{ }_{A}^{B} \mathbf{R}(\varphi)=\left[\begin{array}{cc}
\cos \varphi & -\sin \varphi \\
\sin \varphi & \cos \varphi
\end{array}\right]
$$

$\varphi$ - the angle of rotation between $x$-axes of $A$ and $B$ coordinate systems. By ${ }_{A}^{B} \mathbf{P}(x, y)$ is denoted the vector that represents coordinates of the origin of frame $B$ according to the origin of frame $A$.

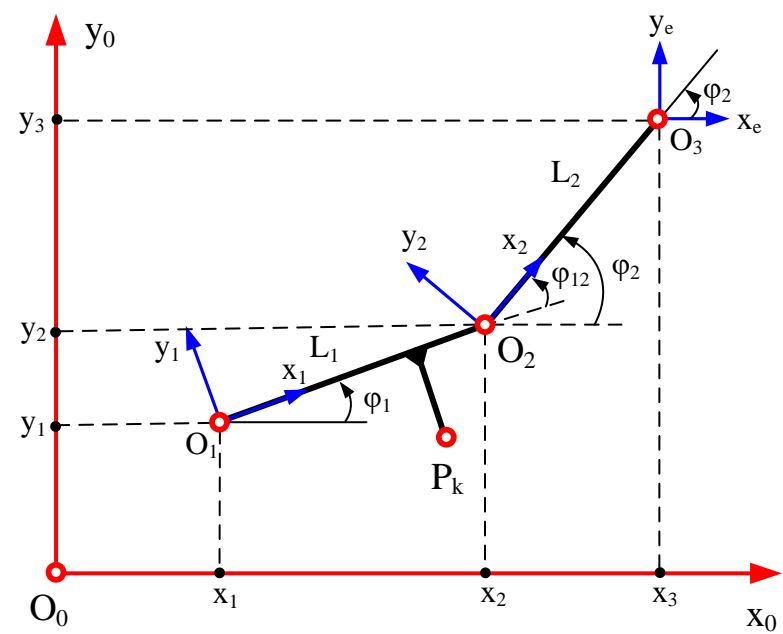

Fig.1 Schematics of RRR dyad

The transformation matrix between $\left\{x_{0} y_{0}\right\}$ and $\left\{x_{e} y_{e}\right\}$ coordinate frames is obtained by a sequential multiplication of a number of transformation matrices between the adjacent frames:

$$
{ }_{0}^{e} \mathbf{T}={ }_{0}^{1} \mathbf{T}\left(\varphi_{1}, x_{1}, y_{1}\right){ }_{1}^{2} \mathbf{T}\left(\varphi_{12}, L_{1}, 0\right){ }_{2}^{e} \mathbf{T}\left(-\varphi_{2}, L_{2}, 0\right)
$$


After the expansion and simplification of Eq. (3) we get:

$$
\left[\begin{array}{ccc}
1 & 0 & x_{3} \\
0 & 1 & y_{3} \\
0 & 0 & 1
\end{array}\right]=\left[\begin{array}{ccc}
1 & 0 & x_{1}+L_{1} \cos \varphi_{1}+L_{2} \cos \varphi_{2} \\
0 & 1 & y_{1}+L_{1} \sin \varphi_{1}+L_{2} \sin \varphi_{2} \\
0 & 0 & 1
\end{array}\right]
$$

Equating the elements $(1,3)$ and $(2,3)$ of the left matrix to the corresponding elements of the right matrix leads to the following position equations:

$$
\mathbf{r}_{1}-\mathbf{r}_{3}=\left[\begin{array}{c}
-L_{1} \cos \varphi_{1}-L_{2} \cos \varphi_{2} \\
-L_{1} \sin \varphi_{1}-L_{2} \sin \varphi_{2}
\end{array}\right]
$$

where by $\mathbf{r}_{1}$ and $\mathbf{r}_{3}$ are denoted the position vectors in the global frame of points $O_{1}$ and $O_{3}, \mathbf{r}_{1}=\left[\begin{array}{ll}x_{1} & y_{1}\end{array}\right]^{T}, \mathbf{r}_{3}=\left[\begin{array}{ll}x_{3} & y_{3}\end{array}\right]^{T}$. In Eq. (3) we also had in mind that

$$
\varphi_{12}=\varphi_{2}-\varphi_{1}
$$

Eqs. (5) constitutes a nonlinear system of transcendental equations with unknown variables $\varphi_{1}$ and $\varphi_{2}$. After elaborate algebraic manipulations are obtained equations for the unknown angles in an explicit form:

$$
\begin{aligned}
& \varphi_{1}=\gamma+p \operatorname{acos}(-C / D) \\
& \varphi_{2}=\operatorname{atan} 2(E, F)
\end{aligned}
$$

where the following notations are used: $A=2 L_{1}\left(x_{1}-x_{3}\right), B=2 L_{1}\left(y_{1}-y_{3}\right), C=L_{1}^{2}-L_{2}^{2}+$ $+\left(x_{1}-x_{3}\right)^{2}+\left(y_{1}-y_{3}\right)^{2}, D=\sqrt{A^{2}+B^{2}}, \gamma=\operatorname{atan} 2(B / D, A / D), E=\left(-\left(y_{1}-y_{3}\right)-L_{1} \sin \varphi_{1}\right) / L_{2}$, $F=\left(-\left(x_{1}-x_{3}\right)-L_{1} \cos \varphi_{1}\right) / L_{2}$. Parameter $p$ specifies the assembly mode of the RRR group and accepts values +1 and -1 .

In addition, the coordinates of inner rotational pair $\mathrm{O}_{2}$ are computed as:

$$
\begin{aligned}
& x_{2}=x_{1}+L_{1} \cos \varphi_{1} \\
& y_{2}=y_{1}+L_{1} \sin \varphi_{1}
\end{aligned}
$$

\subsection{Formulation of the velocity and acceleration equations}

Once the position equations are established, the corresponding velocity and acceleration equations are obtained by a straightforward differentiation with respect to the time. The linear velocities of joints $O_{1}$ and $O_{3}$ and the angular velocities of links $\dot{\varphi}_{1}$ and $\dot{\varphi}_{2}$ are related by Jacobian matrix $\mathbf{J}$ :

$$
\mathbf{V}=\mathbf{J} \Omega
$$

where $\boldsymbol{\Omega}=\left[\begin{array}{ll}\dot{\varphi}_{1} & \dot{\varphi}_{2}\end{array}\right]^{T}$ is the vector of the unknown angular velocities of the links and $\mathbf{V}=\left[\dot{\mathbf{r}}_{1}-\dot{\mathbf{r}}_{3}\right]$ is the vector of the difference of the known linear velocities of the external rotational pairs. The Jacobian for the considered RRR dyad has the following form: 


$$
\mathbf{J}=\left[\begin{array}{cc}
L_{1} \sin \varphi_{1} & L_{2} \sin \varphi_{2} \\
-L_{1} \cos \varphi_{1} & -L_{2} \cos \varphi_{2}
\end{array}\right]
$$

The singular configuration for the dyad is determined from the Eq. (12). For $L_{1}$, $L_{2} \neq 0$ it is easy to find that the determinant (12) vanishes when $\varphi_{1}=\varphi_{2}$ and singularities exist in this particular configuration.

$$
\operatorname{det}(\mathbf{J})=-L_{1} L_{2} \sin \left(\varphi_{1}-\varphi_{2}\right)
$$

The unknown angular velocities are determined from the equation

$$
\boldsymbol{\Omega}=\mathbf{J}^{-1} \mathbf{V}
$$

where by $\mathbf{J}^{-1}$ is denoted the inverse of the Jacobian:

$$
\mathbf{J}^{-1}=\frac{1}{\sin \left(\varphi_{1}-\varphi_{2}\right)}\left[\begin{array}{cc}
\cos \varphi_{2} / L_{1} & \sin \varphi_{2} / L_{1} \\
-\cos \varphi_{1} / L_{2} & -\sin \varphi_{1} / L_{2}
\end{array}\right]
$$

The time differentiation of Eq. (10) leads to

$$
\mathbf{A}=\dot{\mathbf{J}} \mathbf{\Omega}+\mathbf{J} \mathbf{\Omega}
$$

which provides the relationship between the accelerations of external pairs $\mathbf{A}=\left[\ddot{\mathbf{r}}_{1}-\ddot{\mathbf{r}}_{3}\right]$ and the angular accelerations of links $\dot{\boldsymbol{\Omega}}=\left[\begin{array}{ll}\ddot{\varphi}_{1} & \ddot{\varphi}_{2}\end{array}\right]^{T}$. From Eq. (15) we obtain the equations for the angular accelerations of the links:

$$
\dot{\mathbf{\Omega}}=\mathbf{J}^{-1}(\mathbf{A}-\mathbf{J} \mathbf{\Omega})
$$

When we use Eq. (16), we have in mind that

$$
\dot{\mathbf{J}}=\left[\begin{array}{ll}
L_{1} \dot{\varphi}_{1} \cos \varphi_{1} & L_{2} \dot{\varphi}_{2} \cos \varphi_{2} \\
L_{1} \dot{\varphi}_{1} \sin \varphi_{1} & L_{2} \dot{\varphi}_{2} \sin \varphi_{2}
\end{array}\right]
$$

The Cartesian coordinates, velocity and acceleration of internal joint $O_{2}$ are calculated by the Eqs. (18), (19) and (20):

$$
\begin{aligned}
& \mathbf{r}_{2}=\mathbf{r}_{1}+L_{1}\left[\begin{array}{ll}
\cos \varphi_{1} & \sin \varphi_{1}
\end{array}\right]^{T} \\
& \dot{\mathbf{r}}_{2}=\dot{\mathbf{r}}_{1}+L_{1} \dot{\varphi}_{1}\left[\begin{array}{ll}
-\sin \varphi_{1} & \cos \varphi_{1}
\end{array}\right]^{T} \\
& \ddot{\mathbf{r}}_{2}=\ddot{\mathbf{r}}_{1}+L_{1}\left[-\left(\ddot{\varphi}_{1} \sin \varphi_{1}+\dot{\varphi}_{1}^{2} \cos \varphi_{1}\right) \quad\left(\ddot{\varphi}_{1} \cos \varphi_{1}-\dot{\varphi}_{1}^{2} \sin \varphi_{1}\right)\right]^{T}
\end{aligned}
$$

Once the unknown coordinates and angles are obtained, the displacement, velocity and acceleration of every point of the links can be determined. A fixed point $P_{k}$ on body $k$ is located from the origin of local frame $\left\{x_{k} y_{k}\right\}$ by vector $\mathbf{u}_{k}^{P}$ and from the origin of global frame $\left\{x_{0} y_{0}\right\}$ by vector $\mathbf{r}_{k}^{P}, k=1,2$ (see Fig.1). Position $\mathbf{r}_{k}^{P}$, velocity $\dot{\mathbf{r}}_{k}^{P}$ and acceleration $\ddot{\mathbf{r}}_{k}^{P}$ of the point are computed by the following relations: 


$$
\begin{gathered}
\mathbf{r}_{k}^{P}=\mathbf{r}_{k}+{ }_{0}^{k} \mathbf{R}\left(\varphi_{k}\right) \mathbf{u}_{k}^{P} \\
\dot{\mathbf{r}}_{k}^{P}=\dot{\mathbf{r}}_{k}+\dot{\varphi}_{k}{ }_{0}^{k} \mathbf{R}_{\varphi}\left(\varphi_{k}\right) \mathbf{u}_{k}^{P} \\
\ddot{\mathbf{r}}_{k}^{P}=\ddot{\mathbf{r}}_{k}-\dot{\varphi}_{k}^{2 k} \mathbf{R}\left(\varphi_{k}\right) \mathbf{u}_{k}^{P}+\ddot{\varphi}_{k}{ }_{0}^{k} \mathbf{R}_{\varphi}\left(\varphi_{k}\right) \mathbf{u}_{k}^{P}
\end{gathered}
$$

where ${ }_{0}^{k} \mathbf{R}_{\varphi}\left(\varphi_{k}\right)=\left[\begin{array}{cc}-\sin \varphi_{k} & -\cos \varphi_{k} \\ \cos \varphi_{k} & -\sin \varphi_{k}\end{array}\right]$ and ${ }_{0}^{k} \mathbf{R}\left(\varphi_{k}\right)=\left[\begin{array}{cc}\cos \varphi_{k} & -\sin \varphi_{k} \\ \sin \varphi_{k} & \cos \varphi_{k}\end{array}\right]$.

In a similar manner, the kinematic equations for the other four types of dyads are derived. In Figs.2-5 are shown the schematics and closed-form solutions for the rest of the Assur's groups, derived in a similar manner as for the RRR group. For the Assur's groups, containing slider pairs in their kinematic structure, one must take into account that the line of the sliding pair motion is defined by the coordinates of a point and angle, for example, for RRT dyad (see Fig.3) are used coordinates $\left(x_{3}, y_{3}\right)$ and angle $\varphi_{3}$, measured from horizontal $x_{0}$-axis. The velocities and accelerations of the dyad output parameters are calculated according to the following kinematical equations:

$$
\begin{gathered}
\dot{\mathbf{q}}_{\text {out }}=\mathbf{J}^{-1} \dot{\mathbf{q}}_{\text {in }} \\
\ddot{\mathbf{q}}_{\text {out }}=\mathbf{J}^{-1}\left(\ddot{\mathbf{q}}_{\text {in }}-\mathbf{J} \dot{\mathbf{q}}_{\text {out }}\right)
\end{gathered}
$$

whose quantities are shown in Table 1, where the following short notations are used: $c_{1}=\cos \varphi_{1}, s_{1}=\sin \varphi_{1}, c_{3}=\cos \varphi_{3}, s_{3}=\sin \varphi_{3}, c_{\alpha}=\cos \alpha, c_{\alpha 1}=\cos \left(\alpha+\varphi_{1}\right), s_{\alpha 1}=\sin \left(\alpha+\varphi_{1}\right)$, $c_{4}=\cos \varphi_{4}, \quad s_{4}=\sin \varphi_{4}, \quad \Delta \dot{x}_{13}=\dot{x}_{1}-\dot{x}_{3}, \quad \Delta \dot{y}_{13}=\dot{y}_{1}-\dot{y}_{3}, \quad \Delta \ddot{x}_{13}=\ddot{x}_{1}-\ddot{x}_{3}, \quad \Delta \ddot{y}_{13}=\ddot{y}_{1}-\ddot{y}_{3}$, $\Delta \dot{x}_{14}=\dot{x}_{1}-\dot{x}_{4}, \Delta \dot{y}_{14}=\dot{y}_{1}-\dot{y}_{4}, \Delta \ddot{x}_{14}=\ddot{x}_{1}-\ddot{x}_{4}, \Delta \ddot{y}_{14}=\ddot{y}_{1}-\ddot{y}_{4}$.

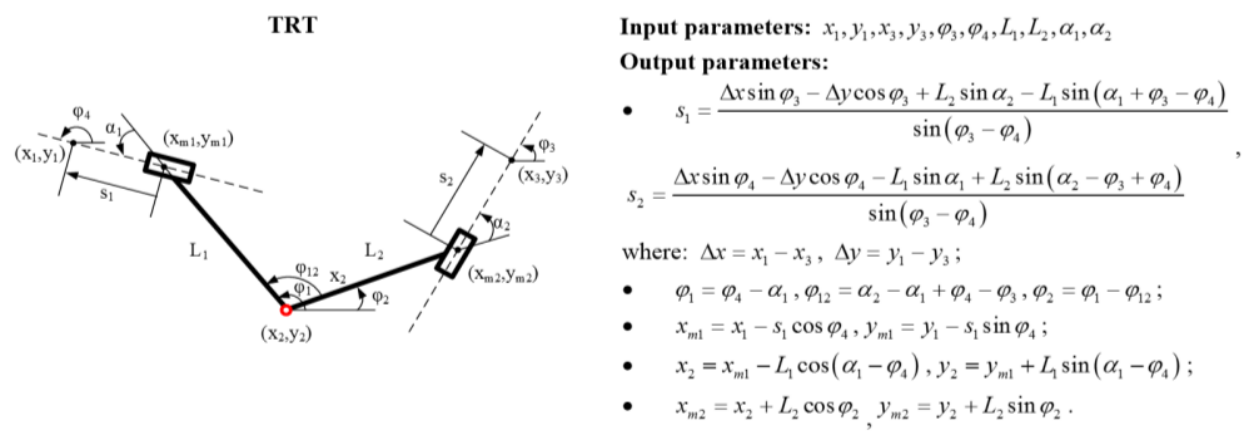

Fig. 2 Schematics and equations for TRT dyad 


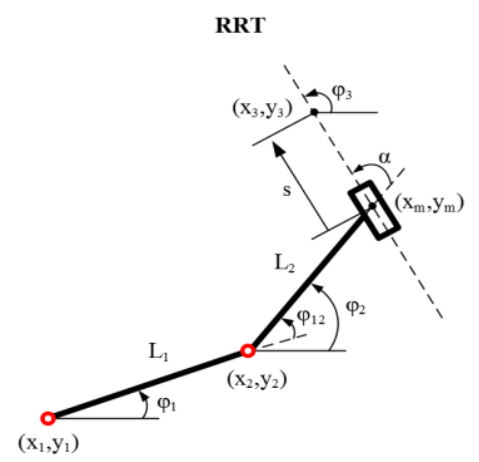

$$
\begin{aligned}
& \text { Input parameters: } x_{1}, y_{1}, x_{3}, y_{3}, L_{1}, L_{2}, \alpha, \varphi_{3}, p \\
& \text { Output parameters: } \\
& \text { - } \quad s=\frac{(-b+p \sqrt{D})}{2 a} \\
& \text { where: } \Delta x=x_{1}-x_{3}, \Delta y=y_{1}-y_{3}, D=b^{2}-4 a c, a=1, \\
& b=2\left(\Delta x \cos \varphi_{3}+\Delta y \sin \varphi_{3}+L_{2} \cos \alpha\right), \\
& c=\Delta x^{2}+\Delta y^{2}+L_{2}^{2}-L_{1}^{2}+2 L_{2}\left(\Delta x \cos \left(\alpha-\varphi_{3}\right)-\Delta y \sin \left(\alpha-\varphi_{3}\right)\right) ; \\
& \text { - } \quad \varphi_{1}=\operatorname{atan} 2\left(\sin \varphi_{1}, \cos \varphi_{1}\right), \\
& \text { where: } \cos \varphi_{1}=\frac{\left(-\Delta x-s \cos \varphi_{3}-L_{2} \cos \left(\alpha-\varphi_{3}\right)\right)}{L_{1}}, \\
& \sin \varphi_{1}=\frac{\left(-\Delta y-s \sin \varphi_{3}+L_{2} \sin \left(\alpha-\varphi_{3}\right)\right)}{L_{1}} ; \\
& \text { - } \quad \varphi_{12}=\varphi_{3}-\varphi_{1}-\alpha ; \varphi_{2}=\varphi_{1}+\varphi_{12} ; \\
& \text { - } x_{2}=x_{1}+L_{1} \cos \varphi_{1}, y_{2}=y_{1}+L_{1} \sin \varphi_{1} ; \\
& \text { - } x_{m}=x_{2}+L_{2} \cos \varphi_{2}, y_{m}=y_{2}+L_{2} \sin \varphi_{2} ;
\end{aligned}
$$

Fig. 3 Schematics and equations for RRT dyad

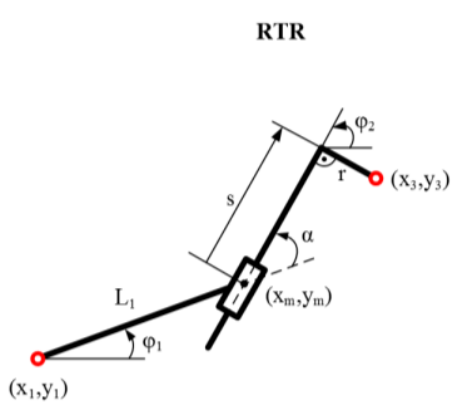

Input parameters: $x_{1}, y_{1}, x_{3}, y_{3}, L_{1}, r, \alpha, p$

Output parameters:

- $s=\frac{(-b+\sqrt{D})}{2 a}$

where: $\Delta x=x_{1}-x_{3}, \Delta y=y_{1}-y_{3}, D=b^{2}-4 a c, a=1$,

$b=2 L_{1} \cos \alpha, c=2 L_{1} r \sin \alpha+L_{1}^{2}+r^{2}-\Delta x^{2}-\Delta y^{2}$,

- $\varphi_{1}=\gamma+p \cdot \operatorname{acos}(-C / D), \varphi_{2}=\varphi_{1}+p \alpha$,

where: $\Delta x=x_{1}-x_{3}, \Delta y=y_{1}-y_{3} \quad A=2 L_{1} \Delta x, B=2 L_{1} \Delta y$,

$C=\Delta x^{2}+\Delta y^{2}+L_{1}^{2}-r^{2}-s^{2}, D=\sqrt{A^{2}+B^{2}}$,

$\gamma=\operatorname{atan} 2(B / D, A / D)$;

- $x_{m}=x_{1}+L_{1} \cos \varphi_{1}, y_{m}=y_{1}+L_{1} \sin \varphi_{1}$

- $x_{2}=x_{m}+s \cos \varphi_{2}, y_{2}=y_{m}+s \sin \varphi_{2}$;

Fig. 4 Schematics and equations for RTR dyad

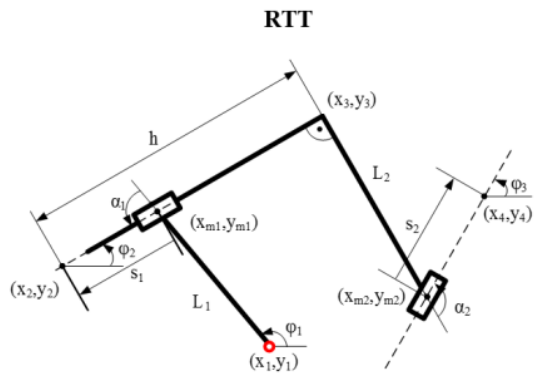

Input parameters: $x_{1}, y_{1}, x_{4}, y_{4}, \varphi_{3}, h, L_{1}, L_{2}, \alpha_{1}, \alpha_{2}$ Output parameters:

$$
\begin{array}{ll}
\text { - } & s_{1}=\frac{\left(\begin{array}{l}
h \cos \alpha_{2}-L_{1} \cos \left(\alpha_{1}+\alpha_{2}\right)-L_{2} \sin \alpha_{2}+ \\
+\left(x_{4}-x_{1}\right) \sin \varphi_{3}+\left(y_{1}-x_{4}\right) \cos \varphi_{3}
\end{array}\right)}{\cos \alpha_{2}}, \\
s_{2}= & \left(\begin{array}{l}
L_{1} \sin \alpha_{1}-L_{2}-x_{1} \cos \left(\alpha_{2}-\varphi_{3}\right)+y_{1} \sin \left(\alpha_{2}-\varphi_{3}\right)+ \\
+x_{4}\left(\cos \left(\alpha_{2}-\varphi_{3}\right)-\sin \left(\alpha_{2}-\varphi_{3}\right)\right)
\end{array}\right) \\
\text { - } \quad \varphi_{1}=\varphi_{3}-\alpha_{1}-\alpha_{2}+3 \pi / 2 ; \\
\text { - } \quad x_{m 1}=x_{1}+L_{1} \cos \varphi_{1}, y_{m 1}=y_{1}+L_{1} \sin \varphi_{1} ; \\
\text { - } \quad x_{2}=x_{m 1}+s_{1} \cos \left(\alpha_{1}+\varphi_{1}\right), y_{2}=y_{m 1}+s_{1} \sin \left(\alpha_{1}+\varphi_{1}\right) ; \\
\text { - } \quad x_{3}=x_{2}-h \cos \left(\alpha_{1}+\varphi_{1}\right), y_{3}=y_{2}-h \sin \left(\alpha_{1}+\varphi_{1}\right) ; \\
\text { - } \quad x_{m 2}=x_{3}-L_{2} \sin \varphi_{1}, y_{m 2}=y_{3}+L_{2} \cos \varphi_{1} ; \\
\text { - } \quad \varphi_{1}=\varphi_{3}-\alpha_{1}-\alpha_{2}+3 \pi / 2, \varphi_{2}=\varphi_{1}+\alpha_{1}-\pi ;
\end{array}
$$

Fig. 5 Schematics and equations for RTT dyad 
Table 1 Velocities and accelerations of the dyads output parameters

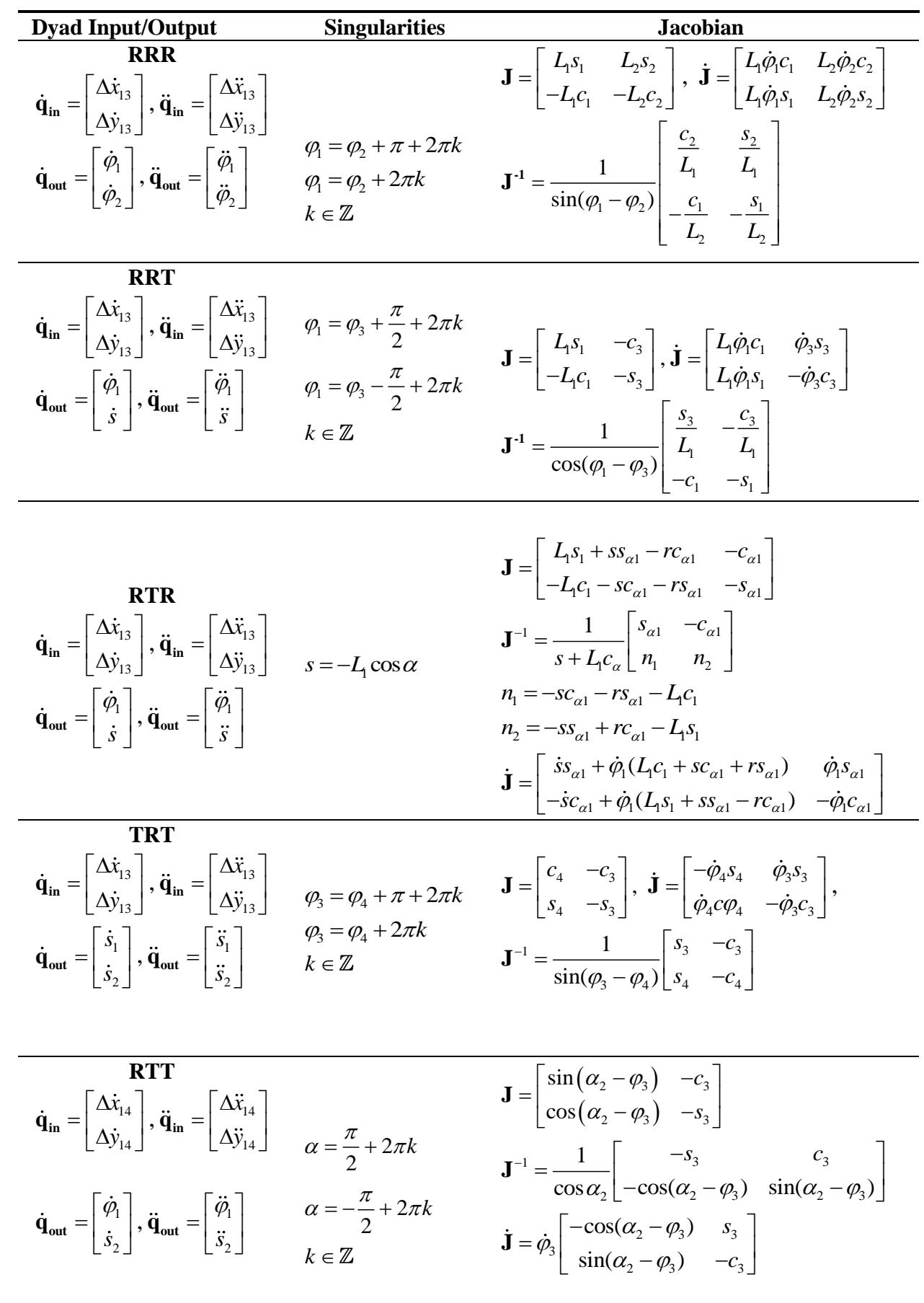




\section{DEVElOPMENT OF AN EXPERIMENTAL ClOUd-BASED EXPERT SySTEM}

In order to combine the modular approach and the optimization synthesis in a common software environment, an experimental cloud-based expert system for synthesis and evolutionary optimization of planar linkages based on the derived in Section 2 Assur's groups equations has been developed and tested. The system consists of the following software modules:

1) A module for linkage synthesis and optimization using evolutionary optimization methods, and,

2) A module with a user interface for linkage visualization, results plotting and animation.

Among the available evolutionary algorithms [23,24] the genetic optimization algorithms (GA) are chosen and implemented in the developed cloud-based expert system [25]. It allows remote creation of the models of genetic algorithms and the receiving of the results by means of a user-friendly interface. By exploiting the application, the user can produce and edit the initial information about the synthesized or optimized linkage; thus he can receive the calculation results as a web page and/or as MS Excel file. The GA framework used technologies and realized functions are shown in Fig.6a).

The sequence of the work with the experimental application is as follows: a) the user creates a description of the specific problem (model of genetic algorithm) that is transported and saved as XML file - Fig. 6b), where the explanation of the purpose of the elements is given by italic letters); b) PHP file reads stored XML and automatically generates a new PHP file for the considered case and the execution is redirected to the generated file; and c) generated PHP file performs the calculations and transmits a report back to the user according to the user requirements.

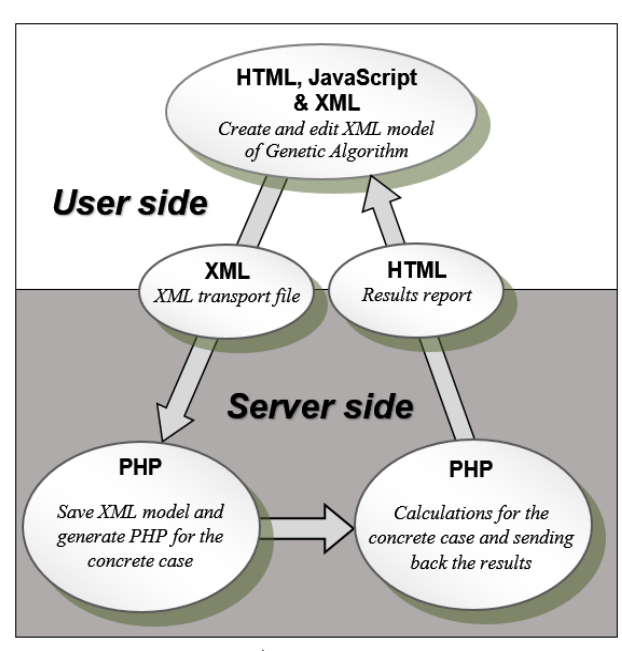

a)

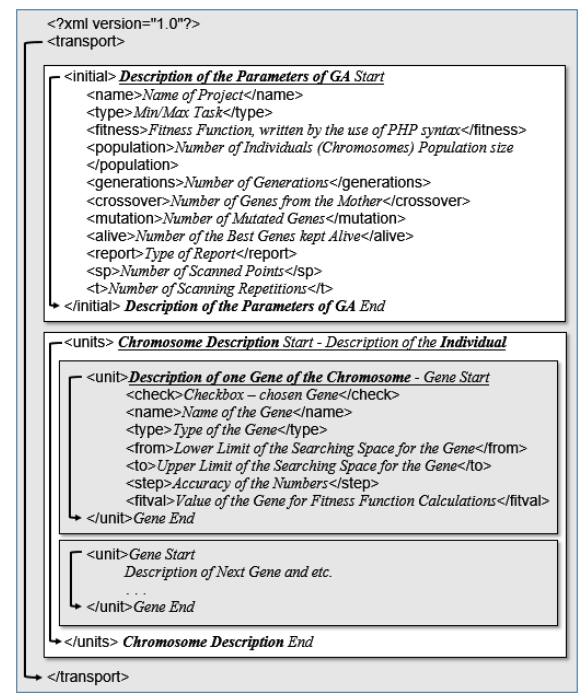

b)

Fig. 6 a) The GA framework; b) Contents of the XML transport file 
The sequence of the steps of genetic algorithms is: a) An initial random population of $n$ chromosomes (solutions) is generated; b) The viability $f(x)$ of each chromosome in the population $n$ (target function - called "fitness function") is calculated; c) The chromosomes are sorted according to their viability (calculated values of the fitness function) and priority for the next population is given to the best $m$ of them $(m<n)$; d) A new population is established by repeating the following steps until the new population is completed, namely preservation of the predetermined number $m$ of the best solutions (according to their fitness - the fitness function values), selection of two parental chromosomes of $m$ chromosomes, use of crossover to cross the parents to form the next generation (children), use of mutation to mutate the newly created chromosomes and paste the new generation in the new population (adding $n-m$ new chromosomes and filling the population); e) loop, go to step b) or stop and return the report if the final check condition is satisfied.

The main novelties, implemented in the developed software system, are:

1) An additional mutation at the beginning of step e) of the best chromosome genes by scanning of every gene within its searching space. Scanning is performed by the use of the dichotomy method [23] following the user-defined requirements for the number of the scanned points (respectively steps) and for the number of the scanning repetitions. If a better solution is found the best chromosome is substituted by the mutated one;

2) The models of GA calculator (parameters, fitness function and chromosome contents) for linkages synthesis and optimization are created by the use of the Assur's groups kinematic equations.

For the results verification by plotting and animation an additional Web-based module is developed with options for editing, design and development of planar linkages. The module is developed by means of free means, namely HTML5, JavaScript and Canvas.

The developed experimental expert system is Web-based and has all advantages of the network computing systems: it overcomes space and time disposition, allows multi-user and multitasking regimes, reduces design costs, etc. Here we have to point to some special outcomes from our research work: chromosome's genes number is not fixed, fitness function is a separate program written in PHP and it is possible to examine the results by the module for visualization and animation. By performing experiments with the obtained results the user can change the parameters of the generated linkages and/or initial parameters of the GA calculator.

\section{NUMERICAL CASE STUDIES AND DISCUSSION}

To test and validate the presented cloud-based expert system two numerical case studies are developed and analyzed. The first case study deals with the dimensional synthesis of a fourbar linkage - Fig.7a) so coupler point $M$ follows the desired path which is an ellipse with a ratio of minor to major semi-axes 1:2 and arbitrary fixed position in the plane. The four-bar linkage is modeled by an RRR dyad, driven by a driver crank. In the second case a Watt I six-bar linkage [26] is considered, composed of two RRR dyads and a driver crank Fig.7b). Coupler point $M$ follows the same ellipse.

For both cases, the optimal values of the design variables are determined so the maximum distance between the desired path, defined by 20 uniformly situated points $P_{\mathrm{i}},(i=1,2, \ldots, 20)$ and 
the calculated coupler point $M_{i}$ curve is minimal. This method can be described by objective function $F$ as follows:

$$
F=\min \left\{\max \Delta r_{i}^{2}\right\},(i=1, \ldots, 20)
$$

where

$$
\Delta r_{i}^{2}=\left(x_{P_{i}}-x_{M_{i}}\right)^{2}+\left(y_{P_{i}}-y_{M_{i}}\right)^{2}
$$

is the squared distance between the points $\left(x_{P_{i}}, y_{P_{i}}\right)$ of the ellipse and the corresponding points $\left(x_{M i}, y_{M i}\right)$ of the coupler. The design variables for the four-bar linkage are $L_{1}, L_{2}, L_{3}$, $L_{4}$ and $\gamma_{1}$-see Fig.4a) and for the six-bar linkage are $L_{1}, L_{2}, L_{3}, L_{4}, L_{5}, L_{6}, L_{7}, L_{8}, \gamma_{1}, \gamma_{2}$ and $\gamma_{3}$ - see Fig.7b). Also, for both linkages the coordinates of points $O$ and $C$ must be determined $-(x O, y O)$ and $(x C, y C)$.

During the synthesis two types of constraints must be satisfied: 1) the design variables (lengths and angles) are changed in predefined limits (see Tables 2 and 3 - Min. and Max. values); 2) the Grashof condition for the $O A B C$ and $B D F E$ four-bar linkages must be satisfied. In addition, to obtain a proportionate from an engineering point of view linkage, additional relations between the links lengths could be defined.

The linkages dimensional synthesis is performed by using a two-stage optimization process. At the initial stage, the stochastic search of the design variables optimal values is performed in a relatively big design variables space. Based on the fitness function change, the design variables values found after 500 generations are considered as optimal for the first stage.

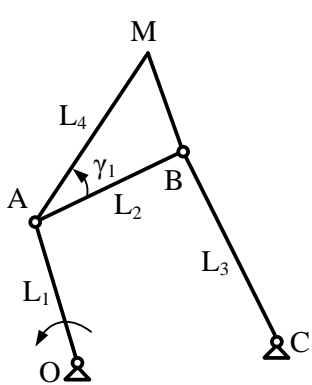

a)

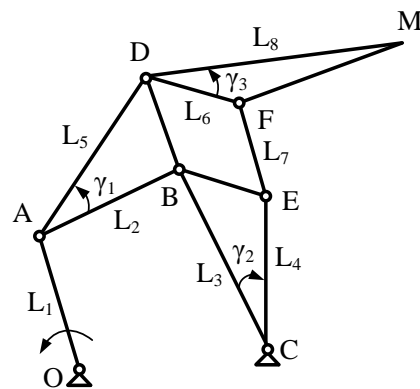

b)

Fig. 7 Schematics of the synthesized linkages: a) four-bar; b) Watt-1 six-bar mechanism

At the second stage, the first stage optimal values are used as initial guesses and the design variables limits are chosen so that the stochastic search is performed in a narrower range of design variables values. In Tables 2 and 3 for the considered linkages the limits of the design variables (dimensions for the lengths are in arbitrary linear units, dimensions for the angles are in degrees) for the two stages are shown. The results for the parameters optimal values and objective function $F_{500}$ after every stage are shown additionally. 
Table 2 Limits of the design variables and optimal values - four-bar linkage

\begin{tabular}{|c|c|c|c|c|c|c|c|c|c|}
\hline & $\mathrm{L}_{1}$ & $\mathrm{~L}_{2}$ & $\mathrm{~L}_{3}$ & $\mathrm{~L}_{4}$ & $\gamma_{1}$ & $\mathrm{xO}$ & $\mathrm{yO}$ & $\mathrm{xC}$ & $\mathrm{yC}$ \\
\hline \multicolumn{10}{|c|}{ Stage 1,500 generations, $F_{500}=2.049$} \\
\hline Min. values & 20 & 20 & 20 & 20 & 0 & -50 & 0 & 0 & -100 \\
\hline Max. values & 100 & 100 & 100 & 100 & 90 & 50 & 100 & 100 & 0 \\
\hline Optimal values & 34.79 & 66 & 80 & 52.35 & 83 & 19.55 & 39.84 & 36.01 & -63 \\
\hline \multicolumn{10}{|c|}{ Stage 2,500 generations, $F_{500}=1.565$} \\
\hline$\overline{\text { Min. }}$ & 30 & 60 & 70 & 40 & 60 & 10 & 30 & 30 & -70 \\
\hline Max. values & 50 & 80 & 90 & 60 & 90 & 30 & 50 & 50 & -50 \\
\hline Optimal values & 37 & 70.96 & 87 & 54 & 73 & 14.48 & 41.89 & 36.65 & -67 \\
\hline
\end{tabular}

Table 3 Limits of the design variables and optimal values - six-bar linkage

\begin{tabular}{lccccccccccccccc}
\hline & $\mathrm{L}_{1}$ & $\mathrm{~L}_{2}$ & $\mathrm{~L}_{3}$ & $\mathrm{~L}_{4}$ & $\mathrm{~L}_{5}$ & $\mathrm{~L}_{6}$ & $\mathrm{~L}_{7}$ & $\mathrm{~L}_{8}$ & $\gamma_{1}$ & $\gamma_{2}$ & $\gamma_{3}$ & $\mathrm{xO}$ & $\mathrm{yO}$ & $\mathrm{xC}$ & $\mathrm{yC}$ \\
\hline Min. values & 20 & 20 & 20 & 20 & 20 & 20 & 20 & 20 & -90 & -90 & -90 & -100 & -100 & -100 & -100 \\
Max. values & 100 & 100 & 100 & 100 & 100 & 100 & 100 & 100 & 90 & 90 & 90 & 100 & 100 & 100 & 100 \\
Optimal values & 32 & 72.99 & 72 & 78 & 85.07 & 71 & 85 & 75 & 89 & -27 & 11 & -30.1 & -6.1 & 44.03 & -81 \\
\hline \multicolumn{10}{c}{ Stage 2,500} & generations, $\mathrm{F}_{500}=1.151$ & & & & & \\
\hline \multicolumn{1}{c}{ Min. values } & 25 & 68 & 68 & 75 & 80 & 65 & 80 & 70 & 85 & -32 & 6 & -35 & -11 & 40 & -85 \\
Max. values & 35 & 78 & 78 & 85 & 90 & 75 & 90 & 80 & 95 & -22 & 16 & -25 & -1 & 50 & -75 \\
Optimal values & 30.95 & 72 & 73 & 85 & 86 & 72 & 85 & 74 & 91 & -24 & 8 & -26.9 & -8.1 & 44 & -85 \\
\hline
\end{tabular}

The presented results show that for the four-bar linkage the improvement of the fitness value at the second stage in relation to the first stage is about $23.6 \%$, while for the six-bar linkage the same value is about $33.2 \%$. In addition, one can see that the fitness value for the six-bar linkage is smaller than the fitness value for the four-bar linkage by $26.4 \%$, i.e. the six-bar linkage better tracks the desired path. This fact is explained by the presence of more geometrical parameters in the six-bar linkage that can be adjusted to minimize the fitness function.

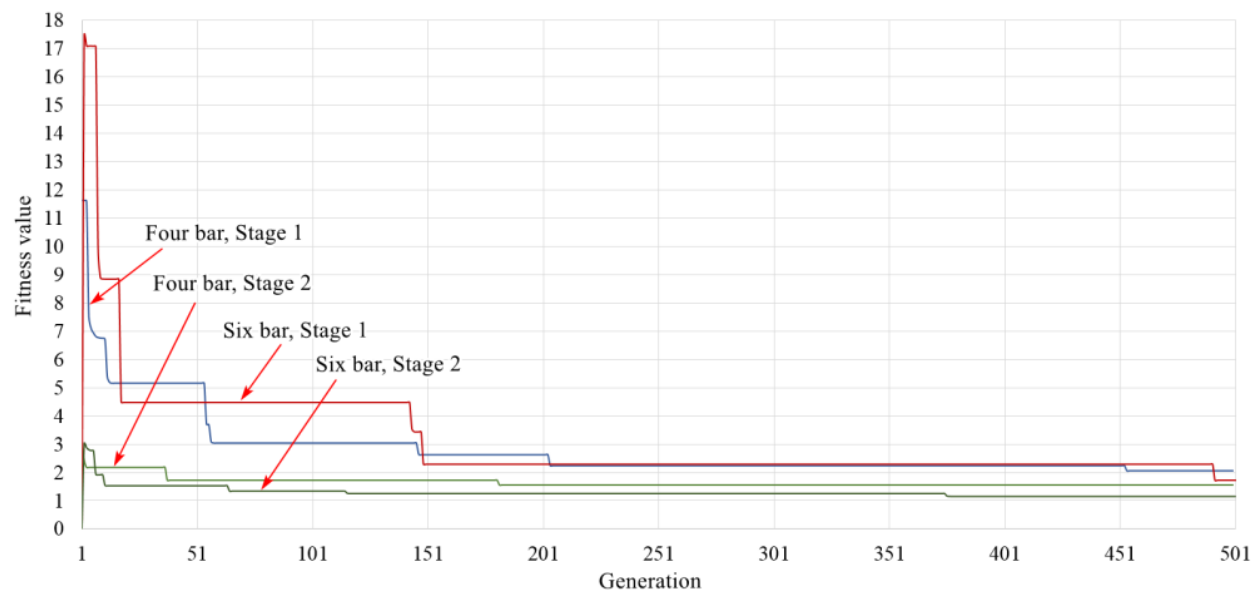

Fig. 8 Evolution of the fitness function values for the considered linkages 
In Fig. 8 the evolution of the fitness function values for the considered case studies is shown. In Figs. 9 and 10 the evolution of the linkages geometrical configurations together with the couplers trajectory and the desired path is shown.

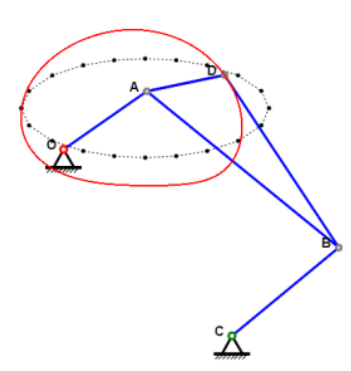

Generation 1, F=11.6161

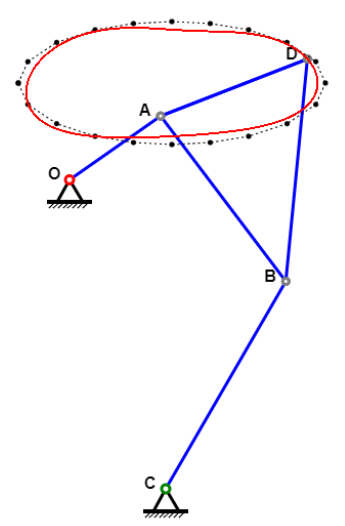

Generation 1, F=2.911277

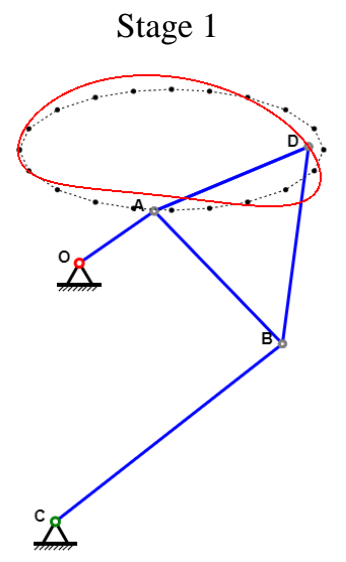

Generation 14, F=5.148866

\section{Stage 2}

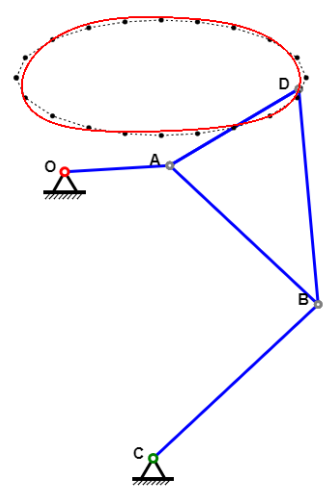

Generation 14, F= 2.181182

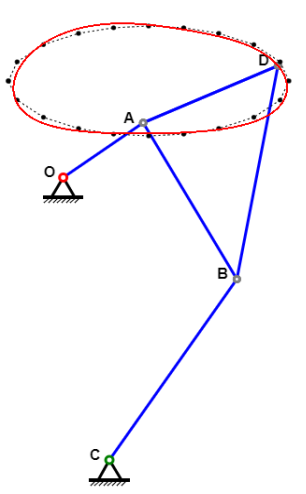

Generation 500, F=2.048843

Fig. 9 Evolution of the four-bar linkage geometrical configurations and the coupler point path 


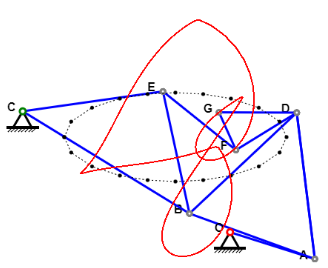

Generation 1, F=17.4918

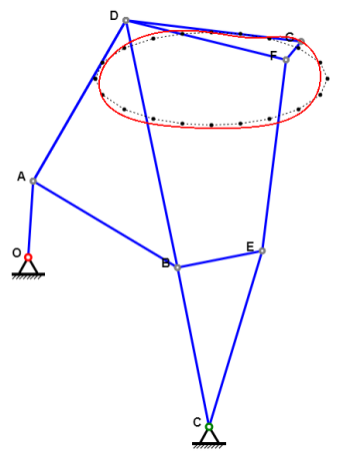

Generation 1, F=3.03592
Stage 1

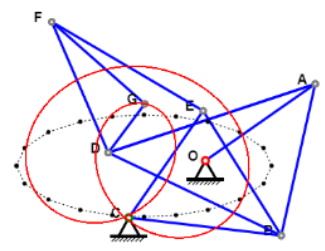

Generation $16, \mathrm{~F}=8.8444$

Stage 2

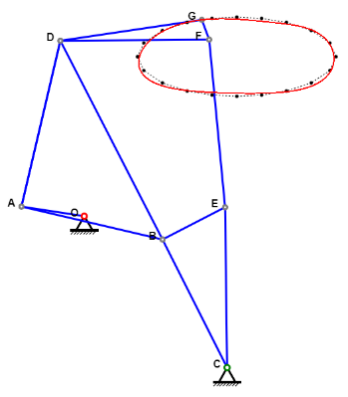

Generation $10, \mathrm{~F}=1.54891$

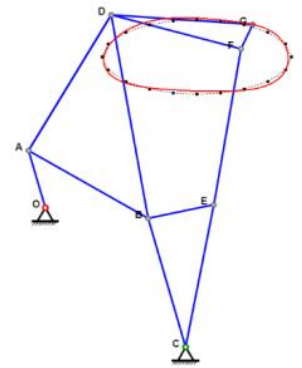

Generation 500, F=1.7238

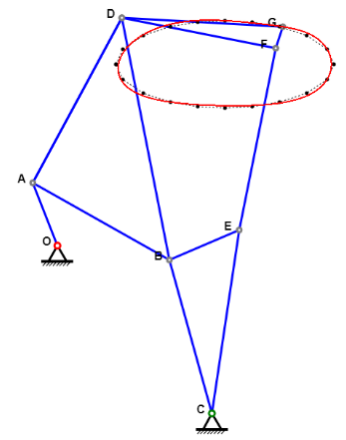

Generation 500, F=1.151466

Fig. 10 Evolution of the six-bar linkage geometrical configurations and the coupler point path

\section{CONCLUSION}

The paper presents a cloud-based expert system for synthesis and evolutionary optimization of planar linkages. The software system allows composition of linkage kinematical structure by means of the modular approach based on Assur's groups. The used approach for obtaining the geometrical relationships between the input and the output variables of the dyads is based on the use of homogeneous transformation matrices. The representations of the dyads as functional blocks with input and output variables allows creating subprograms or modules for each dyad type and utilizing them as building blocks when creating linkages with arbitrary complexity. The velocity and acceleration equations are obtained by a straightforward differentiation with respect to the time and the relationships between the input and output velocities and accelerations are represented by the use of the Jacobian, its inverse and time derivative.

The developed expert system allows a dimensional synthesis of planar linkages by using genetic optimization algorithms. One feature is remote creation of the models of genetic algorithms and the receiving of the results by using a user-friendly interface. By exploiting the application, the user can produce and edit the initial information about the synthesized or optimized linkage; thus he can receive the calculation results as a web page 
and/or as MS Excel file. An additional mutation of the best chromosome genes by scanning of every gene within its searching space improves the optimal solution. If a better solution is found the best chromosome is substituted by the mutated one.

The analyzed numerical case studies show the applicability of the developed software system for mechanism analysis, synthesis and optimization. Because the number of genes is not limited, linkages with a very big number of design variables can be synthesized exploiting the developed approach.

\section{REFERENCES}

1. Artobolevsky, I., 1988, Theory of machines and mechanisms, Moscow, Science, 640 p. (in Russian)

2. Erdman, A., Sandor, G., Kota, S., 2001, Mechanism Design: Analysis and Synthesis, Pearson, 688 p.

3. Marinković, Z., Marinković, D., Petrović, G., Milić, P.,2012, Modelling and simulation of dynamic behaviour of electric motor driven mechanisms, Tehnicki vjesnik, 19(4), pp.717-725.

4. Mitrev, R., Janošević, D., Marinković, D., 2017, Dynamical modelling of hydraulic excavator considered as a multibody system, Tehnicki vjesnik, 24 (Supplement 2), pp.327-338.

5. Working Model User's Guide, 1989, Knowledge Revolution.

6. SAM 7.0 User's Guide, 2014, ARTAS Engineering Software.

7. Ansys Theory Reference, release 5.6, 1999 Ansys Inc.

8. Campbell, M., Cheng, H., 2007, Teaching computer-aided mechanism design and analysis using a high-level mechanism toolkit, Comput. Appl. Eng. Educ., 15(4), pp. 277-288.

9. Katwyk, K., Cheng, H., 1997, XLINKAGE: A Web-based analysis and simulation tool for planar mechanical systems, Proceedings of DETC'97, ASME Design Engineering Technical Conferences, September 14-17, 1997, Sacramento, California

10. Mitrev, R., Tudjarov, B., Kubota, N., 2013, Web based solutions for mechanical engineering, Proc. of the conference IWACIII '2013, Shanghai, China, 18-21 October 2013, pp. GS2-9.1-GS2-9.6.

11. Song, L., Wu, X., Yang, Z., 2008, Research on web-based optimization for path generation synthesis of planar four-bar linkage, IEEE International Conference on Mechatronics and Automation, Takamatsu, pp.1085-1088.

12. Todorov, T., Nikolov, R., 1997, On a Program for Simulation and Optimization of Planar Mechanisms, Proceedings of 11th International Conference "Systems for Automation of Engineering and Research", SAER'97, pp. $156-160$.

13. Hansen, M.R., 1996, A general method for analysis of planar mechanisms using a modular approach, Mechanism and Machine Theory, 31(8), pp. 1155-1166.

14. Galetti, C.U., 1986, A note on modular approaches to planar linkage kinematic analysis, Mechanism and Machine Theory, 21(5), pp.385-391.

15. Fritzson, P., 2003, Principles of Object-Oriented Modeling and Simulation with Modelica 2.1, Wiley, 944 p.

16. Simionescu, P., 2016, MeKin2D: Suite for Planar Mechanism Kinematics, ASME International Design Engineering Technical Conferences and Computers and Information in Engineering Conference, Volume 5B: 40th Mechanisms and Robotics Conference, pp. V05BT07A083.

17. Galletti, C., Giannotti, E., 2009, Assur's-Groups-Based Simulation for Teaching Kinematics of Planar Linkages, Proc.of the 19thCongress of the Italian Society for Theoretical and Applied Mechanics AIMETA, Ancona, Italy.

18. Mesa, L., Durango, S., 2005, Solucion analitica de mecanismos usando grupos de Assur, Scientia et Technica, 11(27), pp.121-126.

19. Popescu, I., Marghitu, D., 2008, Structural design of planar mechanisms with dyads, Multibody Syst Dyn, 19(4), pp. 407-425.

20. Varbanov, H., Yankova, T., Kulev, K., Lilov, S., 2006, S\&A-Expert system for planar mechanisms design, Expert Systems with Applications, 31(3), pp.558-569.

21. Simionescu, P.A., 2014, Computer Aided Graphing and Simulation Tools for AutoCAD Users, CRC Press, $632 \mathrm{p}$.

22. Marghitu, D., Dupac, M., 2012, Advanced Dynamics Analytical and Numerical Calculations with MATLAB, Springer, New York, $610 \mathrm{p}$.

23. Tsonev, S., Vitliemov, V., Koev, P., 2004, Optimization Methods, University of Rousse, Rousse, 2nd ed., 248 p. (in Bulgarian). 
24. Stoven-Dubois, A., Botzheim, J., Kubota, N., 2016, Fuzzy Gesture Expression Model for an Interactive and Safe Robot Partner, Journal of Network Intelligence, 1(4), pp. 119-129.

25. Tudjarov, B., Kubota, N., Penchev, V., Hristov, V., 2011, Web based modeling and calculation of genetic algorithms, International Workshop on Advanced Computational Intelligence and Intelligent Informatics, IWACIII 2011, Suzhou, China.

26. McCarthy J., Soh G., 2011. Geometric Design of Linkages, Springer Verlag, 448 p. 